\title{
Charge asymmetry in electron/positron energy loss in nuclear Bremsstrahlung
}

\author{
Roman N. Lee ${ }^{a}$ and Andrey F. Pikelner ${ }^{b}$ \\ ${ }^{a}$ Budker Institute of Nuclear Physics, \\ Lavrentieva pr. 11, Novosibirsk 630090, Russia \\ ${ }^{b}$ Joint Institute for Nuclear Research, \\ Joliot-Curie, 6, Dubna 141980, Russia \\ E-mail: r.n.lee@inp.nsk.su, pikelner@theor.jinr.ru
}

\begin{abstract}
We calculate the leading Coulomb correction to the Bremsstrahlung energy loss in the electron-nucleus collisions for arbitrary energy of the incoming particle. This correction determines the charge asymmetry, i.e., the difference of electron and positron energy loss. The result is presented in terms of the classical polylogarithms $\mathrm{Li}_{2}$ and $\mathrm{Li}_{3}$. We use modern multiloop methods based on the IBP reduction and on the differential equations for master integrals. We provide both the threshold and the high-energy asymptotics of the found asymmetry and compare them with the available results.
\end{abstract}

KEYWORDS: NLO Computations

ArXiv EPrint: 2109.04926 


\section{Contents}

1 Introduction 1

2 Energy loss 1

3 Methods 2

4 Results $\quad 6$

5 Conclusion 10

A $\mathcal{A}$ and $\mathcal{S}$ functions via classical polylogarithms $\quad 12$

\section{Introduction}

The electron Bremsstrahlung in a nuclear field is one of the most fundamental processes in QED. Its investigation, both theoretical and experimental, has a long history going back to refs. [1-3]. In these papers, the spectrum and the radiation energy weighted cross section have been calculated in the leading Born approximation. The first two papers used the high-energy approximation $\mathcal{E} \gg m$, where $\mathcal{E}$ is the energy of the incoming electron and $m$ is the electron mass. Later on a lot of effort has been devoted to the exact account of the Coulomb corrections and the screening at high energies, $[4,5]$. The first correction in $m / \mathcal{E}$ to the photon spectrum exact in the nucleus charge has been calculated in ref. [6]. This correction extended the applicability of the resulting formula for the spectrum to the region of intermediate energies. However, it seems that, up to the present time, little was known about the Coulomb corrections in the region $\mathcal{E}-m \sim m$. In particular, the leading contribution to the charge asymmetry of the energy loss was never considered before in this energy range, to the best of our knowledge.

The present paper fills this gap by calculating the first Coulomb correction to the energy-weighted cross section. To this end, we apply contemporary multiloop methods based on IBP reduction, differential equations and dimensional recurrence relations. Last but not least, we use this calculation to demonstrate a new technique of obtaining the dimensional recurrence relations for the boundary constants starting from dimensional recurrence relations and differential equations for the original master integrals which depend on parameter.

\section{$2 \quad$ Energy loss}

Energy loss in electron Bremsstrahlung on a nucleus is defined as

$$
\phi=\int \frac{\omega}{\mathcal{E}} d \sigma_{e Z \rightarrow e \gamma Z}=\int \frac{\omega}{\mathcal{E}} \frac{\overline{|M|^{2}}}{2|\boldsymbol{p}|} \frac{d \Phi}{2 \mathcal{E}}, \quad d \Phi=2 \pi \delta\left(\mathcal{E}-\mathcal{E}^{\prime}-\omega\right) \frac{d \boldsymbol{p}^{\prime}}{2 \mathcal{E}^{\prime}(2 \pi)^{3}} \frac{d \boldsymbol{k}}{2 \omega(2 \pi)^{3}},
$$




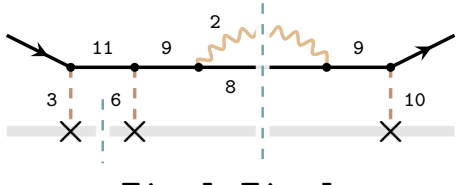

Final-Final

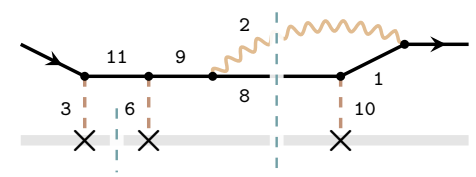

Final-Initial
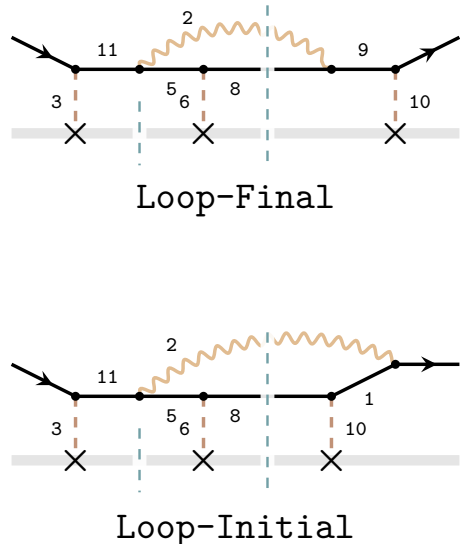

Loop-Initial
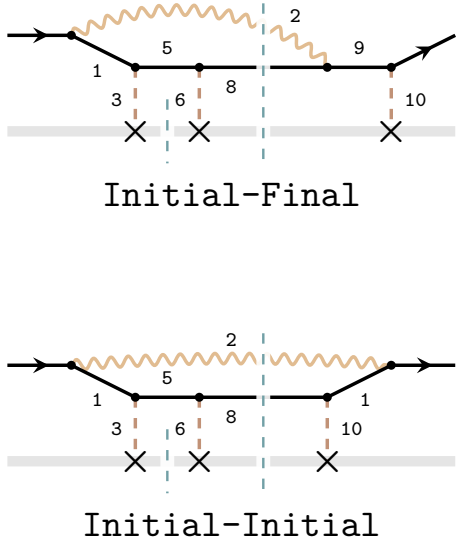

Initial-Initial

Figure 1. Diagrams contributing to NLO cross section.

where $(\mathcal{E}, \boldsymbol{p}),\left(\mathcal{E}^{\prime}, \boldsymbol{p}^{\prime}\right)$, and $(\omega, \boldsymbol{k})$ are the momenta of the incoming electron, scattered electron and emitted photon, respectively, and $\overline{|M|^{2}}$ denotes the square of matrix element averaged/summed over the polarizations of initial/final particles. The physical meaning of $\phi$ is the average fraction of electron energy lost per collision. This quantity plays an important role in the description of the particle propagation in the matter: when multiplied by the density of the scattering centers, it gives the stopping power $S(\mathcal{E})=-d \mathcal{E} / d x$, which in turn defines the radiation length $X_{0}=\int_{0}^{\mathcal{E}} d \mathcal{E}_{1} / S\left(\mathcal{E}_{1}\right)$. It depends on the initial energy $\mathcal{E}$ and the nucleus charge number $Z$. The energy loss of the positron is given by a formal replacement $Z \rightarrow-Z$. When $Z \alpha \ll 1$ (here $Z$ is the charge number of the nucleus), $\phi$ can be expanded in $Z \alpha$ with the leading term $\propto \alpha(Z \alpha)^{2}$. Energy loss in the leading Born approximation has been calculated long ago in Racah paper [3]:

$$
\bar{\phi}_{\mathrm{LO}}=\frac{12 \mathcal{E}^{2}+4 m^{2}}{3 \mathcal{E} p} \ln \frac{\mathcal{E}+p}{m}-\frac{8 \mathcal{E}+6 p}{3 \mathcal{E} p^{2}} \ln ^{2} \frac{\mathcal{E}+p}{m}-\frac{4}{3}-\frac{2}{\mathcal{E} p} \operatorname{Li}_{2}\left(-\frac{2 p(\mathcal{E}+p)}{m^{2}}\right),
$$

where $\bar{\phi} \stackrel{\text { def }}{=} \phi / \sigma_{0}$ with $\sigma_{0} \stackrel{\text { def }}{=} \alpha(Z \alpha)^{2} / m^{2}$.

In order to calculate the charge asymmetry in the leading approximation, one has to account for the first correction with respect to the parameter $Z \alpha$. Let $M=\sum_{k=1}^{\infty} M_{k}$, where the term $M_{k} \propto(Z \alpha)^{k}$ corresponds to the contribution of diagrams with $k$ Coulomb exchanges between electron and nucleus. Then the first $C$-odd correction to $\phi$ is determined by eq. (2.1) with the replacement $|M|^{2} \rightarrow 2 \operatorname{Re}\left[M_{2}^{*} M_{1}\right]$.

\section{Methods}

In order to apply multiloop methods, we use dimensional regularization and express the phase-spaces integrals via loop integrals with cut propagators using the relation

$$
\frac{d^{d-1} \boldsymbol{p}^{\prime}}{2 \mathcal{E}^{\prime}(2 \pi)^{d-1}}=\frac{d^{d} p^{\prime}}{(2 \pi)^{d}} 2 \pi \delta_{+}\left(p^{\prime 2}-m^{2}\right)
$$


Then the contribution of $M_{2}^{*} M_{1}$ is given by the sum of cut diagrams depicted in figure 1. In the limit $\epsilon \rightarrow 0$ the quantity $M_{2}$ is infrared divergent due to the long range nature of the Coulomb interaction. However, this divergence is known to be absorbed into a complex phase factor in the full amplitude. It means that $M_{2}=\frac{1}{\epsilon} a M_{1}+O\left(\epsilon^{0}\right)$ where $a$ is a purely imaginary number, so that $\operatorname{Re}\left[M_{2}^{*} M_{1}\right]$ has a finite limit when $\epsilon \rightarrow 0$. Then, the integration over final particles phase space also has a finite limit when $\epsilon \rightarrow 0$ as the infrared divergencies do not show up due to additional factor $\omega$ under the integral sign.

We consider the following family of integrals

$$
j\left(n_{1}, \ldots, n_{12}\right)=\int \frac{d q d k d p^{\prime}}{i \pi^{3 d / 2}} \prod_{k \in\{1, \ldots, 12\} \backslash C}\left(D_{k}+i 0\right)^{-n_{k}} \times \prod_{k \in C} \frac{\delta^{\left(n_{k}-1\right)}\left(-D_{k}\right)}{\left(n_{k}-1\right) !},
$$

where the set $C=\{2,4,7,8\}$ enumerates the cut denominators

$$
D_{2}=k^{2}, \quad D_{4}=q \cdot n, \quad D_{7}=Q \cdot n, \quad D_{8}=p^{\prime 2} .
$$

where $Q=p-p^{\prime}-k$ and $n=(1, \mathbf{0})$ is the time direction. These cut denominators correspond to the on-shell condition for the emitted photon (2), the zero energy transfer to the heavy nucleus $(4,7)$ and the on-shell condition for the final electron (8). The remaining propagators are

$$
\begin{aligned}
& D_{1}=(p-k)^{2}-m^{2}, \quad D_{3}=q^{2}, \quad D_{5}=(p-k-q)^{2}-m^{2}, \quad D_{6}=(Q-q)^{2}, \\
& D_{9}=\left(k+p^{\prime}\right)^{2}-m^{2}, \quad D_{10}=Q^{2}, \quad D_{11}=(p-q)^{2}-m^{2}, \quad D_{12}=k \cdot n \text {. }
\end{aligned}
$$

All diagrams in figure 1 are expressed via integrals $j\left(n_{1}, \ldots n_{12}\right)$ for which $n_{k \in C}>0$, $n_{12} \leqslant 0$ and at least one of $n_{1}, n_{5}, n_{9}, n_{11}$ is non-positive.

IBP reduction ${ }^{1}$ reveals 61 master integrals. We construct a differential system

$$
\partial_{\mathcal{E}} \boldsymbol{j}=M \boldsymbol{j},
$$

where $\boldsymbol{j}=\left(j_{1}, \ldots j_{61}\right)^{\top}$ is a column of LiteRed master integrals. Then we reduce this system to $\epsilon$-form $[10,11]$ using the Libra package [12]. In order to find the transformation

$$
\boldsymbol{j}=T \boldsymbol{J}
$$

to the canonical basis $\boldsymbol{J}=\left(J_{1}, \ldots, J_{61}\right)$, we pass to the variable $z \in(0,1)$ related to $\mathcal{E}$ via

$$
\mathcal{E}=m \frac{1+z^{2}}{1-z^{2}}, \quad z=\sqrt{\frac{\mathcal{E}-m}{\mathcal{E}+m}} .
$$

Then we have

$$
\partial_{z} \boldsymbol{J}=\epsilon A(z) \boldsymbol{J}=\epsilon\left[\frac{A_{0}}{z}+\frac{A_{1}}{z-1}+\frac{A_{2}}{z+1}+\frac{2 z A_{3}}{z^{2}+1}\right] \boldsymbol{J}
$$

where $A_{n}$ are some constant matrices.

\footnotetext{
${ }^{1}$ IBP reduction was performed using the LiteRed2 package [7-9].
} 
Boundary conditions. We represent the solution as

$$
\boldsymbol{J}(z)=U(z) \boldsymbol{C}
$$

where $U(z)$ is the evolution operator and $C$ is a column of the boundary constants to be fixed. We fix the asymptotics of the evolution operator to be $U(z) \sim z^{\epsilon A_{0}}$, where $A_{0}$ is defined in eq. (3.6). Then, around the point $z=0$, the evolution operator $U(z)$ can be expanded in the generalized power series of the form

$$
\begin{aligned}
U(z)=\sum_{n=0}^{\infty}\{ & U_{n} z^{n}+U_{n+2 \epsilon} z^{n+2 \epsilon}+U_{n-2 \epsilon} z^{n-2 \epsilon}+U_{n-4 \epsilon} z^{n-4 \epsilon}+U_{n-4 \epsilon}^{(1)} z^{n-4 \epsilon} \log z \\
& \left.+U_{n-6 \epsilon} z^{n-6 \epsilon}+U_{n-8 \epsilon} z^{n-8 \epsilon}\right\},
\end{aligned}
$$

where the leading terms $(n=0)$ are simply given by $z^{\epsilon A_{0}}$ and next terms can be found from recurrence relation, as described in ref. [13]. From this expansion and eq. (3.4), it is obvious that the boundary constants $\boldsymbol{C}$ can be determined by fixing a properly chosen set of 61 asymptotic coefficients of master integrals. In order to determine which specific set is suitable, we use the approach described in ref. [14]. Libra has all the necessary tools for finding a column of asymptotic coefficients $c$ required to fix the boundary conditions and the 'adapter' matrix $L$ which maps this column to the column of boundary constants, such that $\boldsymbol{C}=L \boldsymbol{c}$ and

$$
\boldsymbol{J}(z)=U(z) L \boldsymbol{c} .
$$

For the description of these tools, we would refer the reader to ref. [12]. Using Libra, we discover that it is sufficient to determine

- Coefficients in front of $z^{6-8 \epsilon}, z^{6-8 \epsilon}, z^{4-8 \epsilon}, z^{4-8 \epsilon}, z^{4-8 \epsilon}, z^{2-8 \epsilon}$ for the master integrals \#\# 1, 3, 9, 16, 19, 28, respectively;

- 12 coefficients in front of $z^{n-6 \epsilon}(n \in \mathbb{Z})$;

- 43 coefficients in total in front of $z^{n-4 \epsilon}, z^{n-2 \epsilon}, z^{n}$ or $z^{n+2 \epsilon}(n \in \mathbb{Z})$.

Let us explain how we obtain the dimensional recurrence relations for the column of boundary constants. We construct dimensional recurrence relation for the LiteRed master integrals

$$
\boldsymbol{j}(z, \epsilon-1)=R(z, \epsilon) \boldsymbol{j}(z, \epsilon),
$$

where $R(z, \epsilon)$ is some rational matrix. Now from eqs. (3.9) and (3.4) we get

$$
\boldsymbol{j}=\widetilde{T} \boldsymbol{c}, \quad \widetilde{T}=T U(z) L,
$$

and treat $\widetilde{T}$ as a transformation matrix connecting $\boldsymbol{j}$ and the column of coefficients $\boldsymbol{c}$. Then the dimensional recurrence relation for $c$ is given by

$$
\boldsymbol{c}(\epsilon-1)=V(\epsilon) \boldsymbol{c}(\epsilon)
$$


where

$$
V(\epsilon)=\widetilde{T}^{-1}(z, \epsilon-1) R(z, \epsilon) \widetilde{T}(z, \epsilon) .
$$

Note that the left-hand side of eq. (3.13) does not depend on $z$, so the right-hand side should not depend on $z$ also. It is essential that Libra has tools for expanding both $\widetilde{T}^{-1}(\epsilon-1)$ and $\widetilde{T}(\epsilon)$ in generalized power series in $z$. Thus, expanding the right-hand side of eq. (3.13) up to sufficiently high order, we find the exact matrix $V$ entering the dimensional recurrence relation (3.12). Note that the required depth of the expansion of $U$ in $z$ appears to be as high as 11 since the powers $z^{n-8 \epsilon}$ related to the nonzero coefficients in $c$ column change essentially upon the shift $\epsilon \rightarrow \epsilon-1$. Note that the matrix $V$ acquires a block-diagonal form with each block corresponding to a specific $k$ in $z^{n-k \epsilon}$ constants. To summarize the above consideration, we have constructed the exact dimensional recurrence (3.12) for the asymptotic coefficients $\boldsymbol{c}$ starting from the dimensional recurrence for the master integrals $j$ and the generalized power series expansion of the evolution operator $U(z)$, which, in turn, is constructed starting from the differential system (3.6). This approach is quite general and can be used in more involved calculations.

Now we note that the phase space integration is subject to severe restrictions due to $\delta$-functions. As we shall see in a moment, this greatly reduces the number of non-zero coefficients from the above list. We have the following power counting:

$$
p^{\prime} \sim p \sim z, \quad \omega \sim \mathcal{E}-m \sim z^{2}
$$

Thus, we have

$$
d \boldsymbol{p}^{\prime} d \boldsymbol{k} \sim z^{d-1}\left(z^{2}\right)^{d-1}=z^{9-6 \epsilon},
$$

i.e. only powers $z^{n-k \epsilon}$ with $n \in \mathbb{Z}, k \geqslant 6$ survive in the threshold asymptotics. Moreover, the terms $\propto z^{n-6 \epsilon}$ come from the region $|\boldsymbol{q}| \sim z^{0}$ and thorough inspection of the functions $D_{k}$ shows that the contribution of this region vanishes. Therefore, we only have to evaluate the six constants

$$
c_{1}^{6}, \quad c_{3}^{6}, \quad c_{9}^{4}, \quad c_{16}^{4}, \quad c_{19}^{4}, \quad c_{28}^{2},
$$

where $c_{k}^{n}$ denotes the coefficient in front of $z^{n-8 \epsilon}$ in the threshold asymptotics of $k$-th master integral. The first five constants can be calculated exactly in $\epsilon$ using parametric representation in terms of either product of $\Gamma$-functions or hypergeometric functions. However, as the sixth constant, $c_{28}^{2}$, requires a special treatment anyway, we prefer to use the DRA method [15] for all constants in eq. (3.14).

The DRA method requires, in addition to the dimensional recurrence relations (3.12), integral representations from which one can determine analytical properties of $\boldsymbol{c}$ as functions of $\epsilon$. We use the standard expansion by regions method to obtain the integral representations for each nonzero constant. For the most complicated constant $c_{28}^{2}$ we obtain a three-fold integral representation. Using SummerTime [16] and the PSLQ algorithm [17], we obtain the expansions of all six constants from eq. (3.14) in terms of alternating multiple zeta values. 


\section{Results}

We organize the series expansion in $Z \alpha$ for the energy-loss in the following form

$$
\phi=\sigma_{0} \bar{\phi}=\sigma_{0}\left[\bar{\phi}_{\mathrm{LO}}+(Z \alpha) \bar{\phi}_{\mathrm{NLO}}+\mathcal{O}(Z \alpha)^{2}\right] .
$$

Here $\bar{\phi}_{\mathrm{LO}}$ is given by eq. (2.2) and our new result for the NLO correction reads ${ }^{2}$

$$
\begin{aligned}
\bar{\phi}_{\mathrm{NLO}}= & \frac{\pi\left(1-z^{2}\right)}{\left(1+z^{2}\right)}\left(\frac{\left(40-7 z^{2}+3 z^{4}\right)}{12 z}-\frac{84-19 z^{2}+71 z^{4}+59 z^{6}-3 z^{8}}{24 z^{2}\left(1-z^{2}\right)} \mathcal{A}_{1}\right. \\
& +\frac{\left(3+z^{2}\right)\left(1+3 z^{2}\right)\left(1+10 z^{2}+z^{4}\right)}{24 z^{3}\left(1-z^{2}\right)}\left(2 \log 2+\mathcal{S}_{1}\right)+\frac{\left(1-z^{4}\right)\left(1+10 z^{2}+z^{4}\right)}{16 z^{4}} \mathcal{A}_{1,-1} \\
& +\frac{\left(27-8 z^{2}\right)}{6} \mathcal{A}_{2}-\frac{\left(1-z^{2}\right)}{z} \mathcal{A}_{1}^{2}-\frac{\left(1-z^{4}\right)\left(3-26 z^{2}+3 z^{4}\right)}{32 z^{4}} \mathcal{A}_{1} \mathcal{S}_{1} \\
& -\frac{\left(1-z^{4}\right)\left(1-4 z^{2}+z^{4}\right)}{4 z^{4}} \mathcal{A}_{1} \log 2+2 \mathcal{A}_{2} \mathcal{S}_{1}+\frac{\left(1-14 z^{2}+z^{4}\right)}{4 z^{2}}\left(\mathcal{A}_{2,-1}+3 \mathcal{A}_{2,1}\right) \\
& \left.+\frac{\left(1-6 z^{2}+z^{4}\right)}{2 z^{2}}\left(\mathcal{A}_{2} \log 2+\mathcal{A}_{3}\right)+\frac{\left(1-z^{2}\right)^{5}}{64 z^{5}} \mathcal{A}_{1}\left(4 \mathcal{A}_{1} \log 2+\mathcal{A}_{1} \mathcal{S}_{1}-2 \mathcal{A}_{1,-1}\right)\right)
\end{aligned}
$$

where $\mathcal{A}_{\boldsymbol{a}}=H_{\boldsymbol{a}}(z)-H_{\boldsymbol{a}}(-z), \mathcal{S}_{\boldsymbol{a}}=H_{\boldsymbol{a}}(z)+H_{\boldsymbol{a}}(-z), z=\frac{\sqrt{\mathcal{E}-m}}{\sqrt{\mathcal{E}+m}}$ and $H_{\boldsymbol{a}}(z)=H_{a_{1}, \ldots, a_{n}}(z)$ denotes a harmonic polylogarithm (HPL) [18]. All HPLs have the transcendental weight 3 , at most, and can be expressed in terms of classical polylogarithms $\mathrm{Li}_{2}$ and $\mathrm{Li}_{3}$ with complicated argument, see appendix A.

Asymptotics. The non-relativistic and high-energy asymptotics of eq. (4.2) can be compared with the corresponding results known from the literature. In particular, the nonrelativistic limit of the Bremsstrahlung spectrum is known since Sommerfeld's paper [19]. This formula is exact in the parameters $Z \alpha / v, Z \alpha / v^{\prime}$, where $v$ and $v^{\prime}$ are the velocities of the initial and final electron. In contrast, our perturbative result is valid for any $v, v^{\prime}$, but implies that $Z \alpha$ is the smallest parameter, i.e., that $Z \alpha / v \ll 1$. Therefore, we should expand the Sommerfeld spectrum in $Z \alpha / v$ and $Z \alpha / v^{\prime}$, multiply it by $\omega / \mathcal{E}$, and integrate over $\omega$ from zero to $m v^{2} / 2$. For the contribution $\propto(Z \alpha)^{3}$ we obtain

$$
\bar{\phi}_{\mathrm{NLO}} \stackrel{\mathcal{E} \rightarrow m}{\sim} \frac{16 \pi}{3 v}(2 \log 2-1)
$$

which precisely reproduces the asymptotics of our result.

The high-energy limit of our result has the form

$$
\bar{\phi}_{\mathrm{NLO}} \stackrel{\mathcal{E} \gg m}{\sim} \frac{\pi}{24}\left(19 \pi^{2}+84\left(2-\zeta_{3}\right)-60 \pi^{2} \log 2\right)+\frac{\pi^{3}}{2} \log \frac{\mathcal{E}}{m} .
$$

In ref. [6] the spectrum of the high-energy Bremsstrahlung has been calculated exactly in $Z \alpha$. The formula for the spectrum obtained in this ref. implies that the final electron remains ultra-relativistic. Meanwhile, it is easy to establish that the region $\mathcal{E}^{\prime} \sim m$

\footnotetext{
${ }^{2}$ One can find results for the $\bar{\phi}_{\mathrm{NLO}}$ in computer-readable form in the supplementary material.
} 
contribute to the non-logarithmic constant in eq. (4.4). Thus, we can only compare the coefficient in front of the logarithm, boxed in eq. (4.4), which perfectly agrees. Note that the end of the spectrum has been considered in ref. [20], mostly motivated by applications to heavy atoms, $Z \alpha \sim 1$. The result of this paper has the form of slowly convergent sum of nested integrals which is difficult to calculate with sufficient precision in our present restrictions $Z \alpha \ll 1$.

Although our present consideration does not allow us to obtain photon spectrum, we can use the calculated master integrals to obtain results for the cross section integrated with the weight $\omega^{k}$ for $k=1,2, \ldots{ }^{3}$ In particular, we can compare the high- and lowenergy asymptotics these quantities with the corresponding results obtained by integration of the high- and low-energy spectra. It is convenient to consider the following quantity

$$
K^{(n)}(Z, \mathcal{E})=\sigma_{0}\left[K_{\mathrm{LO}}^{(n)}+(Z \alpha) K_{\mathrm{NLO}}^{(n)}+\ldots\right] \stackrel{\text { def }}{=} \int_{0}^{T}(1-\omega / T)^{n} \frac{\omega}{\mathcal{E}} d \sigma_{e Z \rightarrow e \gamma Z}(Z, \mathcal{E}),
$$

where $T=\mathcal{E}-m$ is the kinetic energy of the incoming electron. From ref. [6], by direct integration of the spectrum we obtain for $n>0$

$$
K_{\mathrm{NLO}}^{(n)}=\frac{\pi^{3}(3+2 n)\left(4+9 n+3 n^{2}\right)}{4 n(1+n)(2+n)(3+n)} \frac{m}{\mathcal{E}}+\mathcal{O}\left(\frac{1}{\mathcal{E}^{2}}\right) .
$$

Note that the contribution of the high-frequency end of the spectrum is negligible for positive $n$. Expanding our results for $K_{Z \alpha}^{(n)}$ in the limit $\mathcal{E} \rightarrow \infty$ we find exact agreement with eq. (4.6) for $n=1, \ldots, 16$. This comparison is a strong check on the validity of the procedure, calculated master integrals and performed IBP reduction.

Similarly, we can compare the low-energy asymptotics of $K^{(n)}$ with the corresponding results obtained by a straightforward integration of the Sommerfeld formula expansion,

$$
K_{\mathrm{NLO}}^{(n)}=\frac{8 \pi}{3 v}\left[\frac{(4 n+3) S_{1}(n)}{(n+1)(2 n+1)}-\frac{2 S_{1}(2 n+1)}{n+1}+\frac{4 \log 2}{2 n+1}\right]+\mathcal{O}(v)
$$

where $S_{1}(n)=\sum_{j=1}^{n} \frac{1}{j}$. Again, we find perfect agreement for $n=0, \ldots, 16$.

Numerical results and plots. For the sake of comparison of our results with those available in the literature, we present in figure 2 the electron-positron asymmetry in energy loss defined as

$$
\eta_{\phi}=\frac{\phi(+Z, \mathcal{E})}{\phi(-Z, \mathcal{E})}
$$

as a function of energy. Together with our perturbative results we plot those obtained using the Sommerfeld spectrum [19] and Elwert-Haug interpolation [21].

In order to obtain Sommerfeld and Elwert-Haug cross sections it was crucial to have fast high-precision numerical evaluation of the Gauss hypergeometric function with complex parameters. In our work we use the implementation provided by the multiprecision floating

\footnotetext{
${ }^{3}$ We get the first six moments $(n=0, \ldots, 5)$ by simply making the IBP reduction of the cross section weighted with $\omega^{n}=D_{12}^{n}$. For higher moments the complexity of the IBP reduction gradually increases and we apply instead an approach described in section 4 .
} 


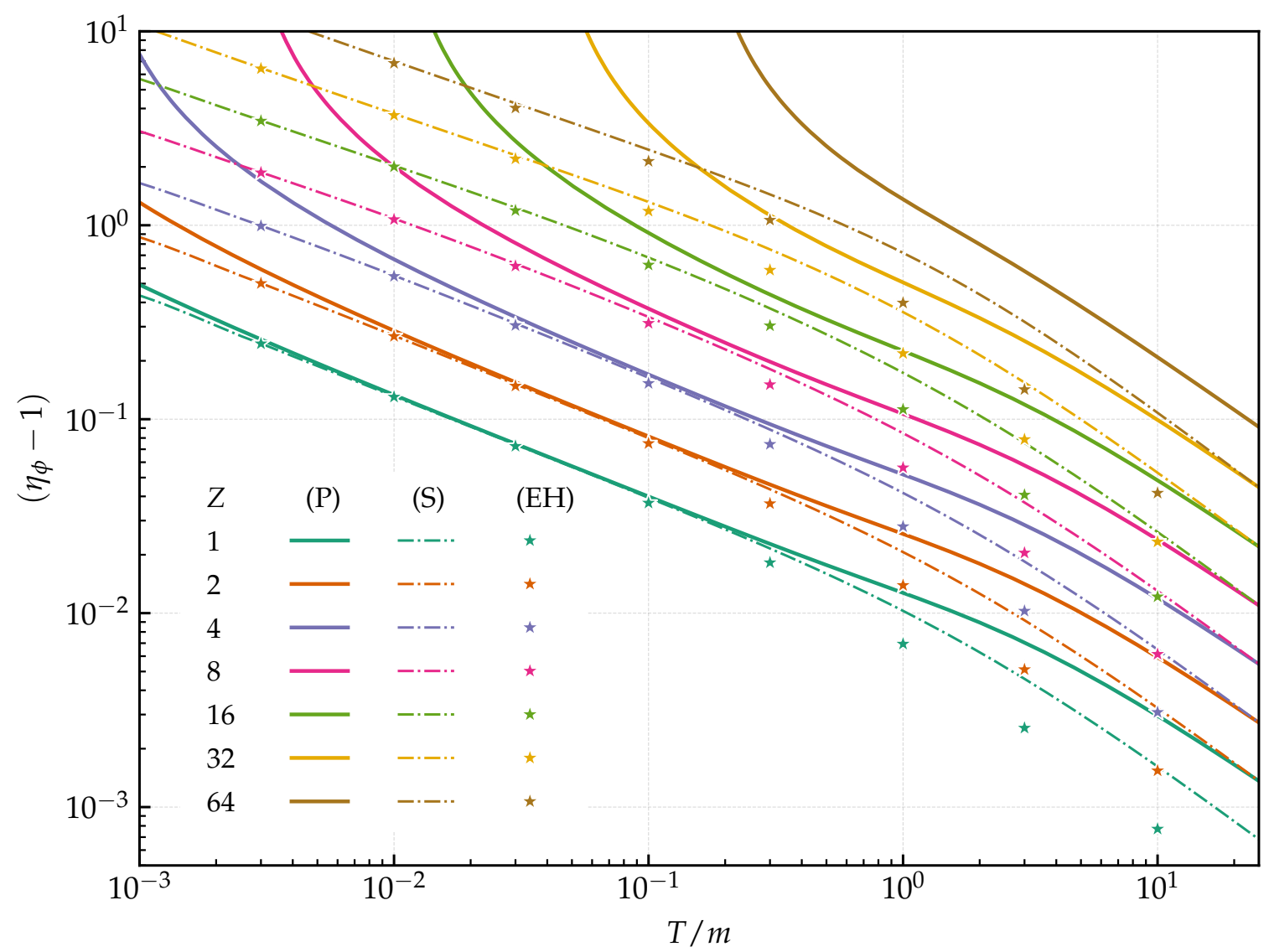

Figure 2. Comparison of the electron to positron energy loss ratio $\eta_{\phi}$ for different $Z$ values. Here $(\mathrm{P})$ is a perturbative NLO result from the current paper and $(\mathrm{S})$ is a non-relativistic exact to all orders in $Z \alpha / v$ result by Sommerfeld [19], $(\mathrm{EH})$ is a result of Elwert-Haug [21].

point library Arb [22]. For the Elwert-Haug cross section we perform in addition the fourdimensional integration of the EH cross section with Cuba library [23]. Of course, since our result is expressed via classical polylogarithms, its numerical evaluation is straightforward.

The deviation of Sommerfeld's result from our result at small enough $T$ even for small $Z$ is not surprising. As we already mentioned our formula does not take into account the corrections of relative order $(Z \alpha / v)^{n}$ for $n \geqslant 2$, thus, when $2 T \lesssim(Z \alpha)^{2}$, Sommerfeld result is reliable, while ours gradually looses its validity as expected.

The deviation of Elwert-Haug result from ours even for small $Z$ at high enough energies can be explained as follows. The original goal of ref. [21] was not to correctly describe the asymmetry, but rather to interpolate between two asymptotics known at that time: the Sommerfeld's result and the Bether-Heitler high-energy asymptotics. The latter is symmetric with respect to the change $Z \rightarrow-Z$, so the Elwert-Haug asymmetry falls off faster than Sommerfeld's. It is interesting to note that our result for the asymmetry goes above the Sommerfeld's.

In ref. [6] the first correction to the Bethe-Maximon spectrum has been calculated. This correction is antisymmetric in $Z$, and thus defines the charge asymmetry in the 


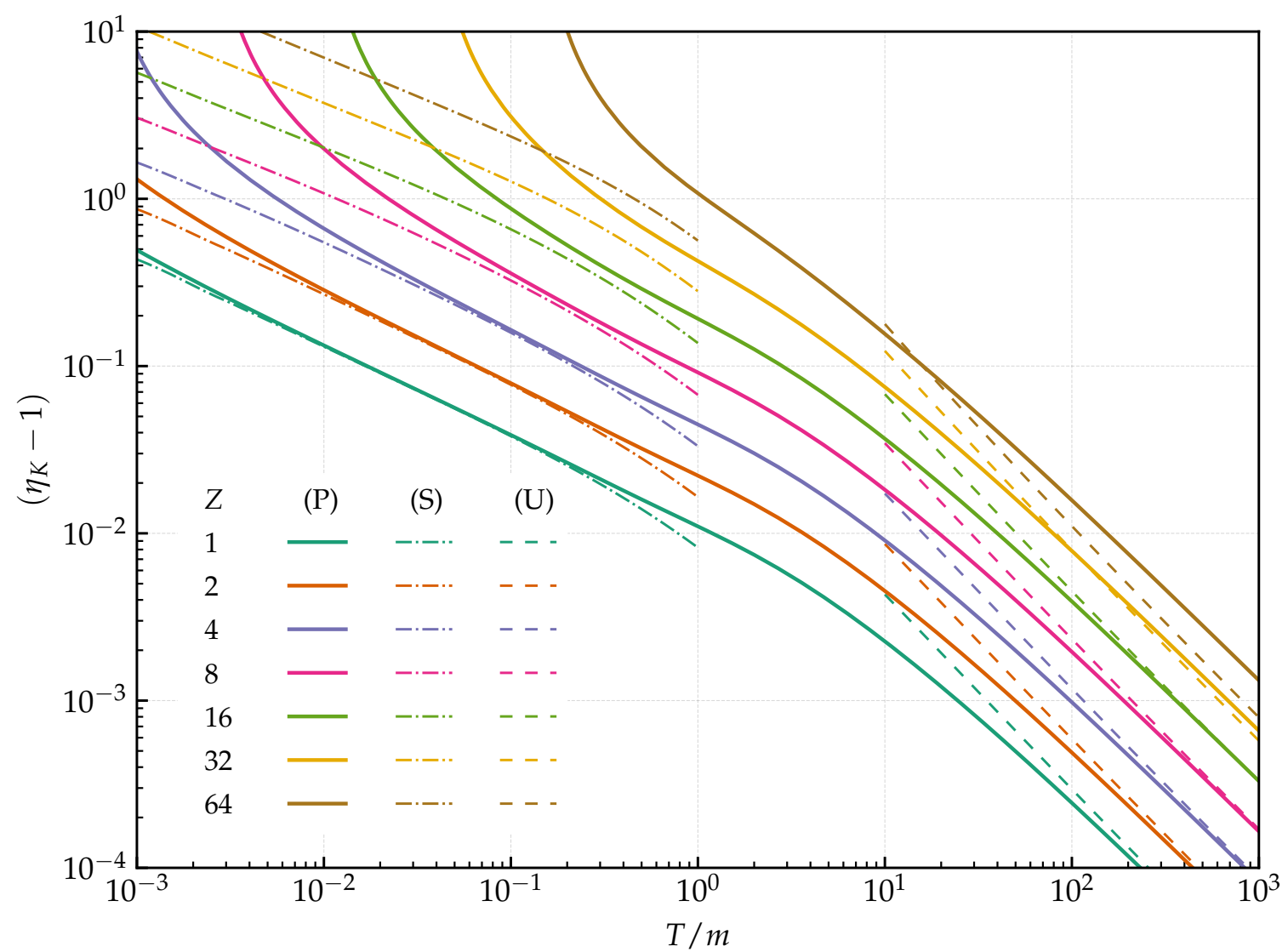

Figure 3. Energy weighted asymmetry, (P) - perturbative NLO result, (S) - Sommerfeld [19], (U) - corrections from [6].

Bremsstrahlung spectrum. However, as explained above, when it concerns energy loss, the spectrum of ref. [6] can be used to obtain it only with logarithmic accuracy, and the extraction of the additive constant from the results of ref. [20] appears to be not practically possible. Due to these reasons, instead of $\eta_{\phi}$ we use a related asymmetry,

$$
\eta_{K}=\frac{K^{(1)}(+Z, \mathcal{E})}{K^{(1)}(-Z, \mathcal{E})}
$$

where $K^{(n)}$ is defined in eq. (4.5). This is asymmetry in the cross section integrated with the weight $\omega \mathcal{E}^{\prime}$ (to be compared with weight $\omega$ for $\phi$ ). In figure 3 we provide a comparison of the asymmetry between positron and electron energy-weighted energy-loss functions $K^{(1)}$ with results for non-relativistic evaluation by Sommerfeld and ultra-relativistic limit predictions from [6]. Our result agrees with the latter at small $Z$ and fails, as expected, when $Z$ becomes large.

Fitting spectrum from fixed moments. As we already mentioned, our approach is suitable for the calculation of the moments, i.e., integrals of the cross section weighted with $\omega^{n}$, or, equivalently, $K^{(n)}$ defined in eq. (4.5). 
Although the computational complexity of the IBP reduction gradually grows with $n$, we have managed to obtain $K_{\mathrm{NLO}}^{(n)}$ for $n=0, \ldots, 5$ rather easily with direct application of IBP reduction tables. In order to obtain yet more moments, we have used the approach based on the derivation of the system of difference equations in variable $n$. To construct this system, we use the fact that the integrals of the family (3.2) which appear in the different moments are the same except the last index $n_{12}$ since $D_{12}=(k \cdot n)=\omega$. Since

$$
\frac{1}{1-t(k \cdot n)}=\sum_{i=0}^{\infty}(k \cdot n)^{i} t^{i}
$$

we can obtain the generating function for the moments by replacing $D_{12}$ with $1-t(k \cdot n)$. Constructing the system of differential equations for integrals with $t$-dependent propagators (4.10) with appropriate ansatz in the form of the series $J=\sum c_{i} t^{i}$ we obtain recurrence relations for the coefficients $c_{i}$ which is nothing but the corresponding moments of original integrals (3.2). On this way, we have calculated moments up to $n=16$. This has provided a number of checks for low- and high-energy asymptotics as explained previously.

Besides, having a considerable number of moments at hand, it is tempting to try to recover the NLO photon spectrum for arbitrary energies. It is convenient to make a fit for the following quantity

$$
\Sigma(z, \tau)=\left(Z \alpha \sigma_{0}\right)^{-1} p \frac{\omega}{\mathcal{E}} \frac{d \sigma_{\mathrm{NLO}}}{d \tau}
$$

where $\tau=\sqrt{T^{\prime} / T}=\sqrt{\frac{\mathcal{E}^{\prime}-m}{\mathcal{E}-m}}$. The convenience of this quantity is that it has finite lowand high- energy limits, known thanks to refs. [19] and [6], respectively. They read

$$
\begin{aligned}
& \Sigma(0, \tau)=\frac{16 \pi}{3}(1-\tau) \log \left(\frac{1+\tau}{1-\tau}\right), \\
& \Sigma(1, \tau)=\frac{\pi^{3}}{2 \tau}\left(\tau^{2}+1\right)\left(2 \tau^{4}-\tau^{2}+2\right) .
\end{aligned}
$$

We tried several fitting bases and found that $\left\{1, \tau, \ldots, \tau^{7}\right\}$ gives the most stable results. ${ }^{4}$ In other words, we write $\Sigma(z, \tau)=\sum_{m=0}^{7} C_{m}(z) \tau^{m}$ and fit coefficients to reproduce the integrals $K_{\mathrm{NLO}}^{(n)}$ with $n=0, \ldots, 7$. Given that $p K_{\mathrm{NLO}}^{(n)}(z)=\int_{0}^{1} d \tau \Sigma(z, \tau) \tau^{2 n}$, we can write the coefficients as

$$
C_{m}(z)=\frac{2 z}{1-z^{2}} \sum_{n=0}^{7}\left(T^{-1}\right)_{m n} K_{\mathrm{NLO}}^{(n)}
$$

where $T=\left\{T_{n m}\right\}=\left\{(2 n+m+1)^{-1}\right\}$.

The resulting spectra are shown in figure 4 .

\section{Conclusion}

To summarize, we have calculated the first Coulomb correction to the energy loss of electron due to Bremsstrahlung in the field of a nucleus for arbitrary electron energy. This correction leads to the asymmetry in the energy loss of an electron and a positron. We have compared

\footnotetext{
${ }^{4}$ Inclusion of yet higher moments into the fit seems to introduce some artifacts in the spectrum.
} 


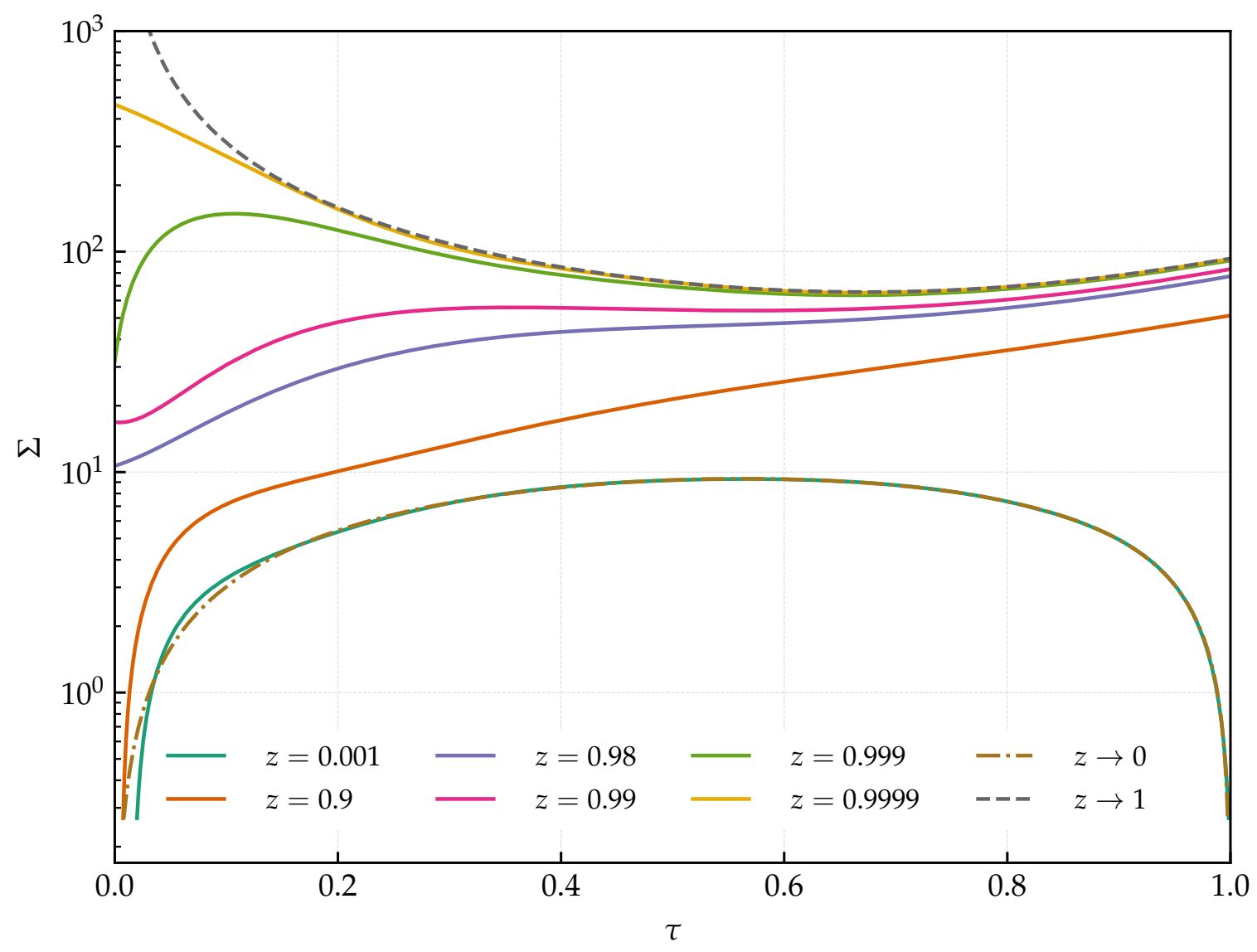

Figure 4. Function $\Sigma(z, \tau)$ for several values of $z=\frac{\sqrt{\mathcal{E}-m}}{\sqrt{\mathcal{E}+m}}$ and its comparison with low energy (dash-dotted curve) and high energy (dashed curve) asymptotics.

the high- and the low-energy asymptotics with available asymptotics and found a perfect agreement. We have calculated also a number of moments of the cross section, as defined in eq. (4.5). The low- and high-energy asymptotics of these moments perfectly agrees with the known results which provides a stringent test of the consistency of our approach. Besides, we have used the calculated moments to approximately recover the photon spectrum, which might be interesting from the point of view of some applications. It would be interesting to compare our fit with direct calculation of the spectrum once/if the latter will be available at some point.

From the perspective of developing new methods, we have demonstrated how to obtain the dimensional recurrence relations for the boundary constants starting from the equations for original master integrals and used this technique to apply the DRA method [15] to the calculation of the constants.

\section{Acknowledgments}

This work was supported by Russian Science Foundation, grant 20-12-00205. The authors wish to thank V.V. Bytev, who participated in the early stages of this work. R.L. is grateful to A.I. Milstein for useful discussions. 


\section{A $\mathcal{A}$ and $\mathcal{S}$ functions via classical polylogarithms}

$$
\begin{aligned}
\mathcal{S}_{1}= & -\log (1-z)-\log (1+z) \\
\mathcal{A}_{1}= & -\log (1-z)+\log (1+z), \\
\mathcal{A}_{2}= & \operatorname{Li}_{2}(z)-\operatorname{Li}_{2}(-z) \\
\mathcal{A}_{3}= & \operatorname{Li}_{3}(z)-\operatorname{Li}_{3}(-z) \\
\mathcal{A}_{1,-1}= & (\log (1+z)-\log (1-z)) \log 2+\operatorname{Li}_{2}\left(\frac{1-z}{2}\right)-\operatorname{Li}_{2}\left(\frac{1+z}{2}\right) \\
\mathcal{A}_{2,1}= & \frac{1}{6}\left(\log ^{3}(1+z)-\log ^{3}(1-z)\right)-\operatorname{Li}_{2}(z) \log (1-z)+\operatorname{Li}_{2}(-z) \log (1+z) \\
& +\operatorname{Li}_{3}(z)-\operatorname{Li}_{3}(-z)-\operatorname{Li}_{3}\left(\frac{z}{1+z}\right)+\operatorname{Li}_{3}\left(\frac{-z}{1-z}\right) \\
\mathcal{A}_{2,-1}= & \left(\frac{\pi^{2}}{12}-\frac{\log ^{2} 2}{2}\right)(\log (1+z)-\log (1-z)) \\
& +\left(\log ^{2}(1+z)-\log { }^{2}(1-z)\right) \frac{\log 2}{2}+\operatorname{Li}_{2}(z) \log (1+z)-\operatorname{Li}_{2}(-z) \log (1-z) \\
& +\operatorname{Li}_{3}\left(\frac{1-z}{2}\right)-\operatorname{Li}_{3}\left(\frac{1+z}{2}\right)-\operatorname{Li}_{3}\left(\frac{-2 z}{1-z}\right)+\operatorname{Li}_{3}\left(\frac{2 z}{1+z}\right) \\
& -\operatorname{Li}_{3}(z)+\operatorname{Li}_{3}(-z)-\operatorname{Li}_{3}\left(\frac{z}{1+z}\right)+\operatorname{Li}_{3}\left(\frac{-z}{1-z}\right)
\end{aligned}
$$

Open Access. This article is distributed under the terms of the Creative Commons Attribution License (CC-BY 4.0), which permits any use, distribution and reproduction in any medium, provided the original author(s) and source are credited.

\section{References}

[1] W. Heitler and F. Sauter, Stopping of fast particles with emission of radiation and the birth of positive electrons, Nature 132 (1933) 892.

[2] H. Bethe and W. Heitler, On the stopping of fast particles and on the creation of positive electrons, Proc. Roy. Soc. Lond. A 146 (1934) 83.

[3] G. Racah, Sopra l'irradiazione nell'urto di particelle veloci (in Italian), Nuovo Cim. 11 (1934) 461.

[4] H.A. Bethe and L.C. Maximon, Theory of Bremsstrahlung and pair production. 1. Differential cross section, Phys. Rev. 93 (1954) 768 [INSPIRE].

[5] H. Olsen and L.C. Maximon, Photon and electron polarization in high-energy Bremsstrahlung and pair production with screening, Phys. Rev. 114 (1959) 887 [INSPIRE].

[6] R.N. Lee, A.I. Milstein, V.M. Strakhovenko and O.Y. Schwarz, Coulomb corrections to Bremsstrahlung in electric field of heavy atom at high energies, J. Exp. Theor. Phys. 100 (2005) 1 [Zh. Eksp. Teor. Fiz. 127 (2005) 5] [hep-ph/0404224] [INSPIRE].

[7] R.N. Lee, Presenting LiteRed: a tool for the Loop InTEgrals REDuction, arXiv:1212.2685 [INSPIRE]. 
[8] R.N. Lee, LiteRed 1.4: a powerful tool for reduction of multiloop integrals, J. Phys. Conf. Ser. 523 (2014) 012059 [arXiv:1310.1145] [INSPIRE].

[9] R.N. Lee, Litered2, to be published soon.

[10] J.M. Henn, Multiloop integrals in dimensional regularization made simple, Phys. Rev. Lett. 110 (2013) 251601 [arXiv:1304.1806] [INSPIRE].

[11] R.N. Lee, Reducing differential equations for multiloop master integrals, JHEP 04 (2015) 108 [arXiv:1411.0911] [INSPIRE].

[12] R.N. Lee, Libra: a package for transformation of differential systems for multiloop integrals, Comput. Phys. Commun. 267 (2021) 108058.

[13] R.N. Lee, A.V. Smirnov and V.A. Smirnov, Solving differential equations for Feynman integrals by expansions near singular points, JHEP 03 (2018) 008 [arXiv: 1709.07525] [INSPIRE].

[14] R.N. Lee, A.V. Smirnov, V.A. Smirnov and M. Steinhauser, Four-loop quark form factor with quartic fundamental colour factor, JHEP 02 (2019) 172 [arXiv:1901.02898] [INSPIRE].

[15] R.N. Lee, Space-time dimensionality D as complex variable: calculating loop integrals using dimensional recurrence relation and analytical properties with respect to D, Nucl. Phys. B 830 (2010) 474 [arXiv:0911.0252] [INSPIRE].

[16] R.N. Lee and K.T. Mingulov, Introducing SummerTime: a package for high-precision computation of sums appearing in DRA method, Comput. Phys. Commun. 203 (2016) 255 [arXiv: 1507.04256] [INSPIRE].

[17] H. Ferguson, D. Bailey and S. Arno, Analysis of PSLQ, an integer relation finding algorithm, Math. Comput. 68 (1999) 351.

[18] E. Remiddi and J.A.M. Vermaseren, Harmonic polylogarithms, Int. J. Mod. Phys. A 15 (2000) 725 [hep-ph/9905237] [INSPIRE].

[19] A. Sommerfeld, Über die Beugung und Bremsung der Elektronen (in German), Annalen Phys. 403 (1931) 257.

[20] A. Di Piazza and A.I. Milstein, High-energy electron-positron photoproduction cross section close to the end of the spectrum, Phys. Rev. A 82 (2010) 042106.

[21] G. Elwert and E. Haug, Calculation of Bremsstrahlung cross sections with Sommerfeld-Maue eigenfunctions, Phys. Rev. 183 (1969) 90.

[22] F. Johansson, Arb: efficient arbitrary-precision midpoint-radius interval arithmetic, IEEE Trans. Comput. 66 (2017) 1281.

[23] T. Hahn, Concurrent Cuba, Comput. Phys. Commun. 207 (2016) 341 [INSPIRE]. 